\title{
Structure-Reactivity Relationships on Substrates and Inhibitors of the Lysine Deacylase Sirtuin 2 from Schistosoma Mansoni (SmSirt2)
}

Daria Monaldi, Dante Rotili, Julien Lancelot, Martin Marek, Nathalie Wössner, Alessia Lucidi, Daniela Tomaselli, Elizabeth Ramos-Morales, Christophe Romier, Raymond J. Pierce, Antonello Mai, Manfred Jung Submitted date: 02/08/2019 - Posted date: 02/08/2019

Licence: CC BY-NC-ND 4.0

Citation information: Monaldi, Daria; Rotili, Dante; Lancelot, Julien; Marek, Martin; Wössner, Nathalie; Lucidi, Alessia; et al. (2019): Structure-Reactivity Relationships on Substrates and Inhibitors of the Lysine Deacylase Sirtuin 2 from Schistosoma Mansoni (SmSirt2). ChemRxiv. Preprint.

The only drug for treatment of Schistosomiasis is Praziquantel, and the possible emergence of resistance makes research on novel therapeutic agents necessary. Targeting of Schistosoma mansoni epigenetic enzymes, which regulate the parasitic life cycle, emerged as promising approach. Due to the strong effects of human Sirtuin inhibitors on parasite survival and reproduction, Schistosoma sirtuins were postulated as therapeutic targets. In vitro testing of synthetic substrates of S. mansoni Sirtuin 2 (SmSirt2) and kinetic experiments on a myristoylated peptide demonstrated lysine long chain deacylation as an intrinsic SmSirt2 activity for the first time. Focused in vitro screening of the GSK Kinetobox library and structure-activity relationships (SAR) of identified hits, led to the first SmSirt2 inhibitors with activity in the low micromolar range. Several SmSirt2 inhibitors showed potency against both larval schistosomes (viability) and adult worms (pairing, egg laying) in culture without general toxicity to human cancer cells.

File list (2)

smSirt2 manuscript revision final.pdf (0.99 MiB)

view on ChemRxiv - download file

Supporting informations revision smSirt2 final.pdf (806.99 KiB)

view on ChemRxiv - download file 


\section{Supporting information}

Structure-reactivity relationships on substrates and inhibitors of the lysine deacylase Sirtuin 2 from Schistosoma mansoni (SmSirt2)

Daria Monaldi ${ }^{1}$, Dante Rotili ${ }^{2 *}$, Julien Lancelot ${ }^{3}$, Martin Marek $^{4}$, Nathalie Wössner ${ }^{1}$, Alessia

Lucidi $^{2}$, Daniela Tomaselli ${ }^{2}$, Elizabeth Ramos-Morales ${ }^{4}$, Christophe Romier ${ }^{4}$, Raymond J. Pierce ${ }^{3}$, Antonello Mai $^{2^{*}}$ and Manfred Jung ${ }^{1^{*}}$

${ }^{1}$ Institute of Pharmaceutical Sciences, University of Freiburg, Albertstr. 25, 79104, Freiburg, Germany.

${ }^{2}$ Dipartimento di Chimica e Tecnologie del Farmaco “Sapienza” Università di Roma, 00185 Rome, Italy

${ }^{3}$ Université de Lille, CNRS, Inserm, CHU Lille, Institut Pasteur de Lille, U1019 - UMR 8204 CIIL - Centre d’Infection et d’Immunité de Lille, 59000 Lille, France

${ }^{4}$ Département de Biologie Structurale Intégrative, Institut de Génétique et Biologie Moléculaire et Cellulaire (IGBMC), Université de Strasbourg (UDS), CNRS, INSERM, 67404 Illkirch Cedex, France

* corresponding authors, E-mail : dante.rotili@uniroma1.it, antonello.mai@uniroma1.it, manfred.jung@pharmazie.uni-freiburg.de 
Table S1. Conversion of ZMAL (1) analogues 3a-g by SmSirt2 ............................................S3

Table S2. Conversion of ZMAL (1) analogues 3a-g by hSirt2 ................................................S3

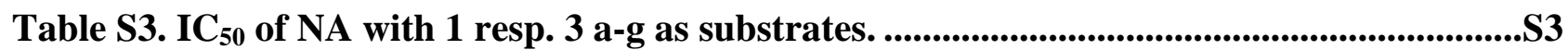

Table S4. Source of the intermediate compounds 45a-e, r, v, w and 46a-h. ............................S3

Table S5. Sequence identities and similarities between smSirT2 and hSirT2.........................S5

Table S6. Effects of 7 and its analogues on schistosomula viability. ............................................S

Table S7. Molecular properties for selected compounds................................................................SZ

$\begin{array}{lc}\text { HPLC Data } & \text { S8 }\end{array}$

$\begin{array}{ll}\text { References } & \text { S13 }\end{array}$ 
Table S1. Conversion of ZMAL (1) analogues 3a-g by SmSirt2.

\begin{tabular}{|c|cccccccc|}
\cline { 2 - 9 } \multicolumn{1}{l|}{ SmSirt2 } & $\mathbf{1}$ & 3a & 3b & 3c & 3d & 3e & 3f & 3g \\
\hline Conversion (\%) $^{a}$ & $21.2 \pm 1.0$ & $63.7 \pm 3.0$ & $91.1 \pm 1.2$ & $89.5 \pm 1.5$ & $75.1 \pm 4.0$ & $58.6 \pm 2.9$ & $27.2 \pm 1.2$ & $13.2 \pm 1.0$ \\
\hline
\end{tabular}

${ }^{a}$ Errors are reported as standard deviation (SD, $\left.\mathrm{n}=3\right)$.

Table S2. Conversion of ZMAL (1) analogues 3a-3g by hSirt2.

\begin{tabular}{|c|cccccccc|}
\cline { 2 - 9 } \multicolumn{1}{c|}{ hSirt2 } & $\mathbf{1}$ & 3a & 3b & 3c & 3d & 3e & 3f & 3g \\
\hline Conversion (\%) $^{\boldsymbol{a}}$ & $28.1 \pm 2.0$ & $77.3 \pm 1.3$ & $79.5 \pm 0.4$ & $28.5 \pm 1.3$ & $47.7 \pm 4.1$ & $9.5 \pm 3.4$ & $12.6 \pm 2.2$ & $2.6 \pm 1.4$ \\
\hline
\end{tabular}

${ }^{a}$ Errors are reported as standard deviation (SD, $\left.\mathrm{n}=3\right)$.

Table S3. $\mathrm{IC}_{50}$ of NA with 1 resp. 3 a-g as substrates.

\begin{tabular}{|l|cccccccc|}
\cline { 2 - 9 } \multicolumn{1}{c|}{} & $\mathbf{1}$ & 3a & 3b & 3c & 3d & 3e & 3f & 3g \\
\hline $\left.\mathbf{I C}_{\mathbf{5 0}} \mathbf{(} \mathbf{\mu M}\right)^{\boldsymbol{a}}$ & $23.1 \pm 1.8$ & $16.3 \pm 2.0$ & $20.5 \pm 0.9$ & $25.8 \pm 2.0$ & $36.1 \pm 3.5$ & $47.9 \pm 2.1$ & $\begin{array}{c}420 \pm 1.16 \\
11.6\end{array}$ & $\begin{array}{l}1600 \pm \\
0.1140\end{array}$ \\
\hline
\end{tabular}

${ }^{a}$ Errors are represented as standard error of the mean (SEM, $\mathrm{n}=3$ ).

\begin{tabular}{|c|c|c|}
\hline \multicolumn{2}{|c|}{ Table S4. Source of the intermediate compounds 45a-e, r, v, wand 46a-h } \\
\hline Compd & Structure & Reference \\
\hline $45 a$ & commercial \\
\hline $45 b$ & commercial \\
\hline 45 & & commercial \\
\hline
\end{tabular}




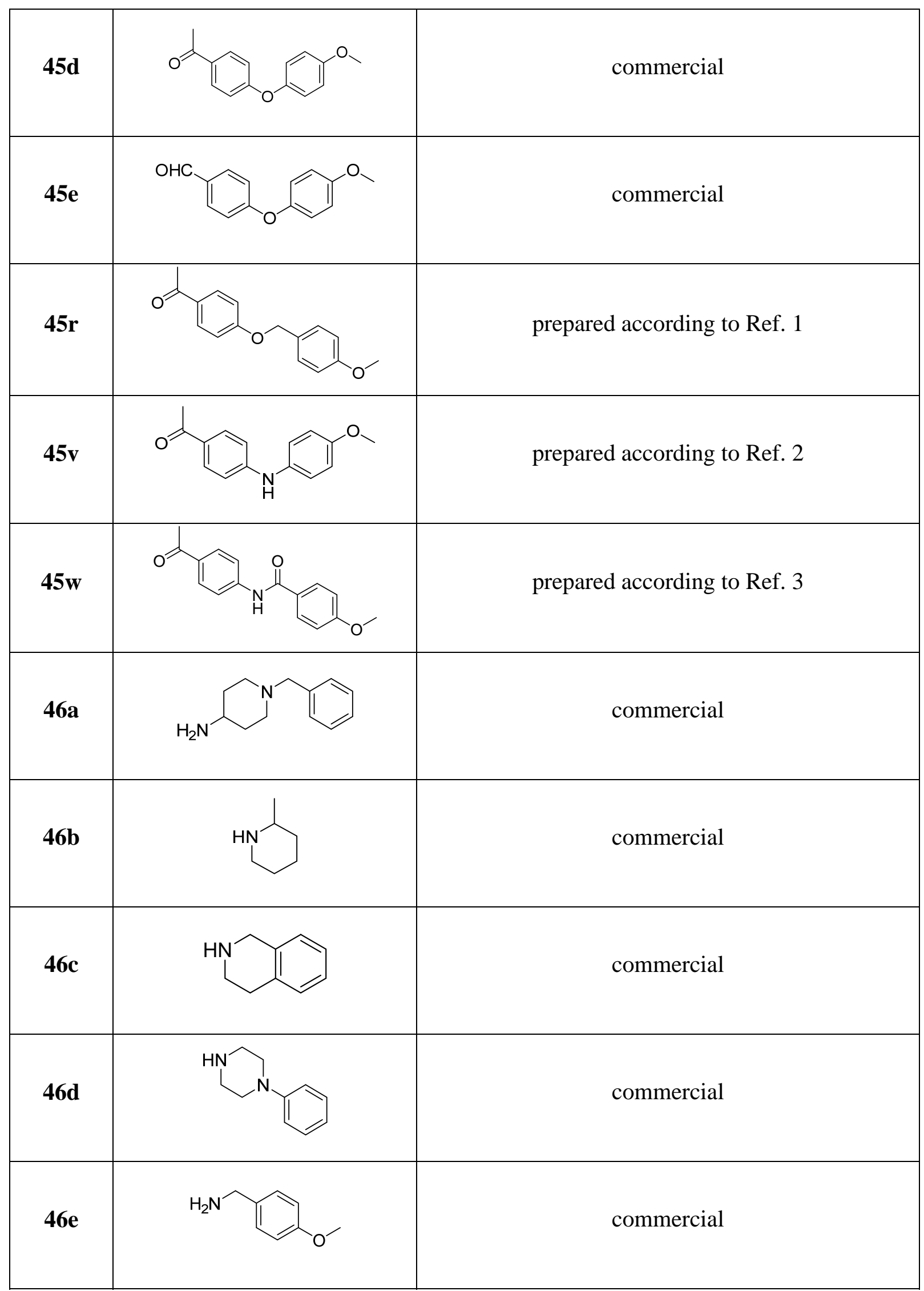




\begin{tabular}{|c|c|c|}
\hline $\mathbf{4 6 f}$ & commercial \\
\hline $\mathbf{4 6 g}$ & $\mathbf{4}$ & commercial \\
\hline $\mathbf{4 6 h}$ & & commercial \\
\hline
\end{tabular}

Table S5. Sequence identities and similarities between smSirT2 and hSirT2

\begin{tabular}{|l|l|l|}
\hline & Full-length & Catalytic domain \\
\hline Identity & $37 \%$ & $44 \%$ \\
\hline Similarity & $49 \%$ & $58 \%$ \\
\hline
\end{tabular}

Phylogeny and sequence similarities between SirT2 from various organisms have already been published. ${ }^{4,5}$ Table $\mathrm{X}$ summarizes sequence identities and similarities calculated between human and S. mansoni SirT2 sequences. For these calculations, both full length and only catalytic domain (based on PDB structure 5D7O) sequences have been used. Both enzymes show identities/similarities indicative of a common fold but with differences that should enable the design of selective inhibitors for smSirT2. 
Table S6. Effects of 7 and its analogues on schistosomula viability.

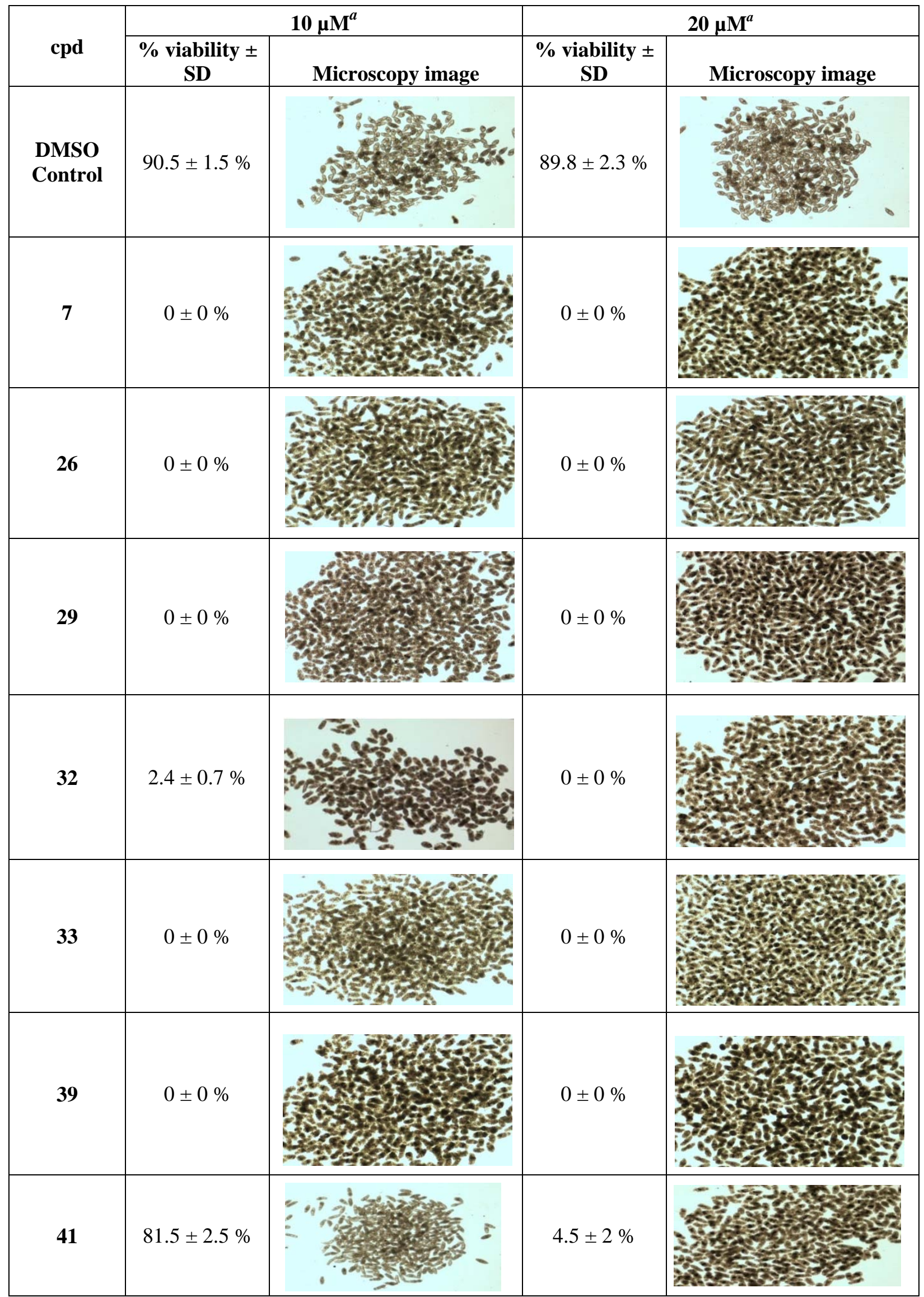


${ }^{a}$ Results obtained by microscopy examination. 72 h, 500 schistosomula/well $(n=2) ; 2$ biological replicates.

Table S7. Molecular properties for selected compounds.

\begin{tabular}{|c|c|c|c|c|c|c|}
\hline Cpd & $M W$ & $c \log P$ & H-Acceptors & H-Donors & TPSA & Druglikeness \\
\hline 4 & 339.825 & 3.7126 & 4 & 1 & 48.99 & 2.4775 \\
\hline 5 & 422.502 & 4.0942 & 6 & 1 & 71.26 & 3.0543 \\
\hline 6 & 417.472 & 2.7956 & 9 & 2 & 125.3 & 3.5505 \\
\hline 7 & 417.472 & 2.7956 & 9 & 2 & 125.3 & 3.5505 \\
\hline 29 & 479.542 & 4.2247 & 9 & 2 & 125.3 & 1.633 \\
\hline 32 & 447.498 & 2.7256 & 10 & 2 & 134.53 & 3.5505 \\
\hline 33 & 509.568 & 4.1547 & 10 & 2 & 134.53 & 1.633 \\
\hline 41 & 463.565 & 3.1511 & 9 & 2 & 150.6 & 3.8621 \\
\hline 42 & 525.635 & 4.5802 & 9 & 2 & 150.6 & 2.0163 \\
\hline
\end{tabular}




\section{HPLC}

3a

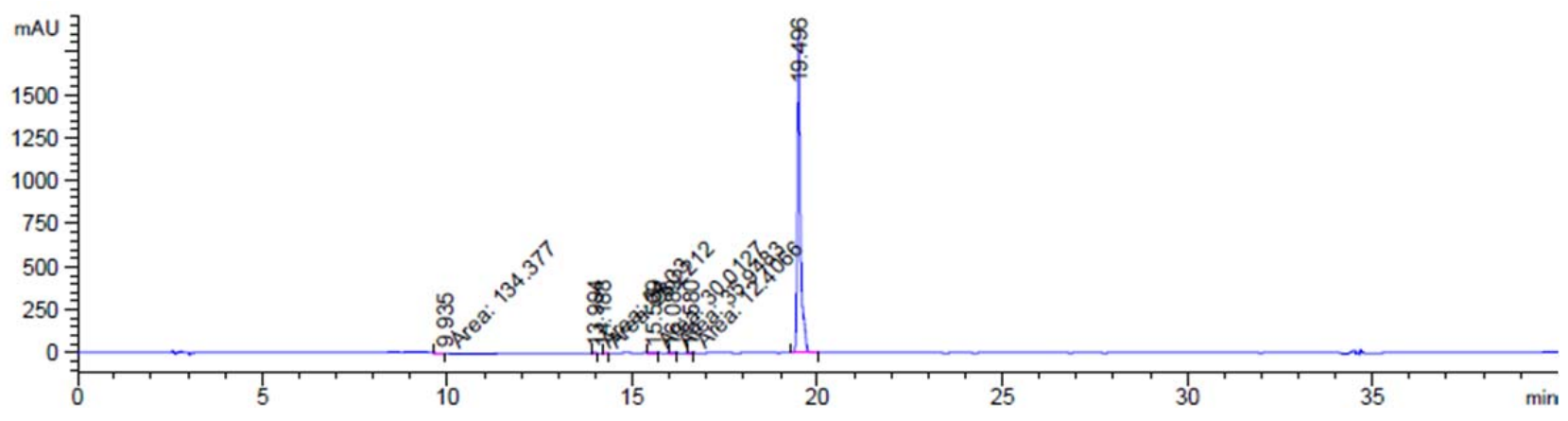

\begin{tabular}{|c|c|c|}
\hline Peak & Retention time (min) & Area (\%) \\
\hline 1 & 9.935 & 1.1476 \\
\hline 2 & 13.994 & 0.4614 \\
\hline 3 & 14.188 & 0.2239 \\
\hline 4 & 15.569 & 0.2563 \\
\hline 5 & 16.089 & 0.3070 \\
\hline 6 & 16.580 & 0.1060 \\
\hline 7 (cp 3a) & 19.496 & 97.4978 \\
\hline
\end{tabular}

$3 b$

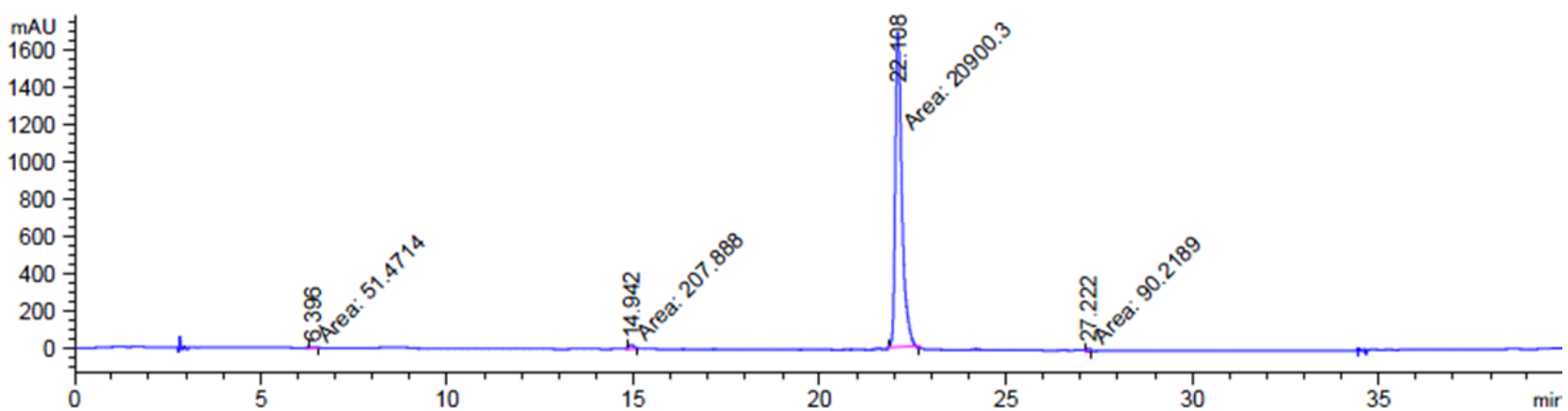




\begin{tabular}{|c|c|c|}
\hline Peak & Retention time (min) & Area (\%) \\
\hline 1 & 6.396 & 0.2422 \\
\hline 2 & 14.942 & 0.9783 \\
\hline 3 (ср 3b) & 22.108 & 98.3549 \\
\hline 4 & 27.222 & 0.4246 \\
\hline
\end{tabular}

3c

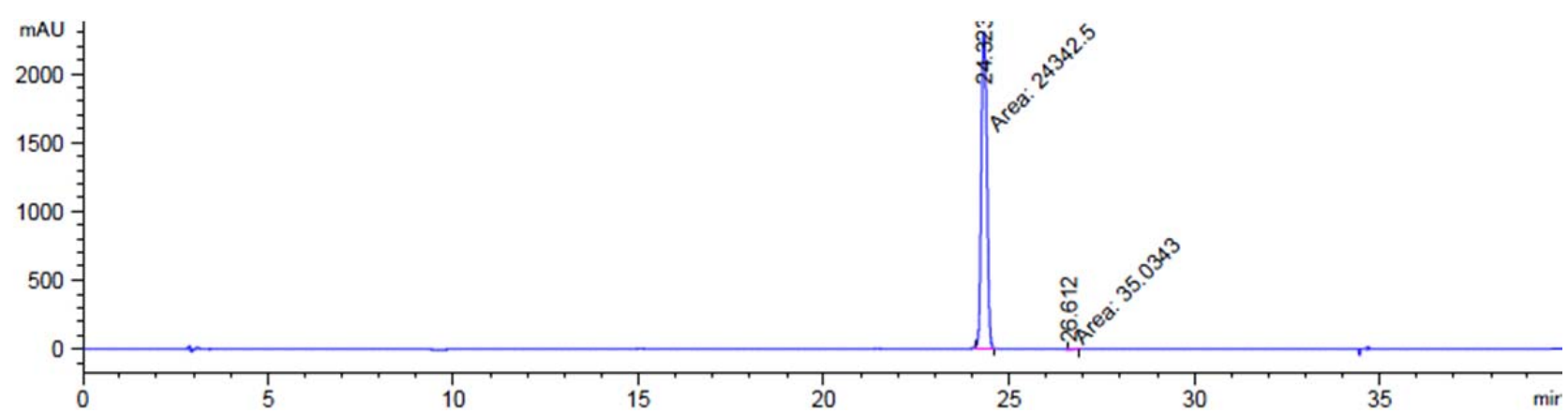

\begin{tabular}{|c|c|c|}
\hline Peak & Retention time (min) & Area (\%) \\
\hline 1 ( ср 3c) & 24.323 & 99.8563 \\
\hline 2 & 26.612 & 0.1437 \\
\hline
\end{tabular}

3d

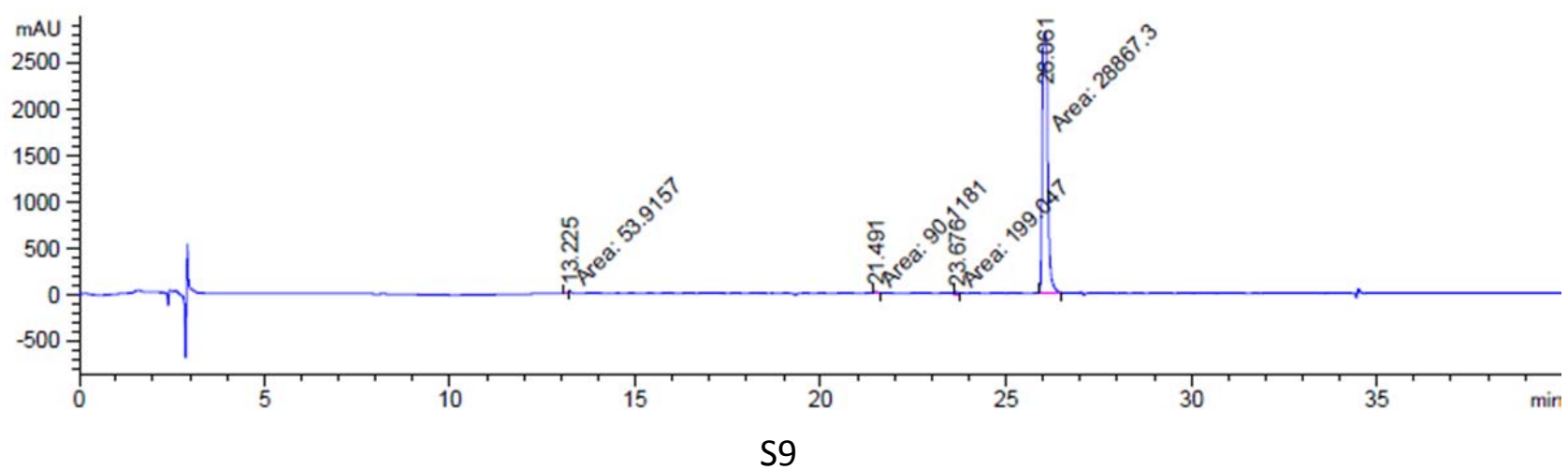




\begin{tabular}{|c|c|c|}
\hline Peak & Retention time (min) & Area (\%) \\
\hline 1 & 13.225 & 0.1846 \\
\hline 2 & 21.491 & 0.3085 \\
\hline 3 & 23.676 & 0.6814 \\
\hline 4 (ср 3d) & 26.061 & 98.8255 \\
\hline
\end{tabular}

3e

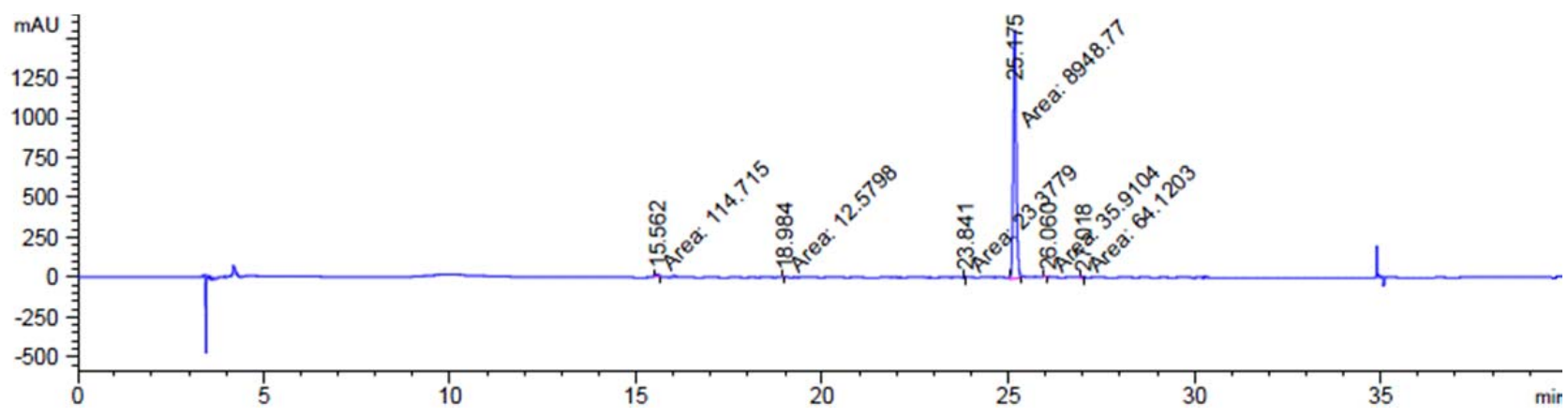

\begin{tabular}{|c|c|c|}
\hline Peak & Retention time (min) & Area (\%) \\
\hline 1 & 15.562 & 1.2470 \\
\hline 2 & 18.984 & 0.1367 \\
\hline 3 & 23.841 & 0.2541 \\
\hline 4 (ср 3e) & 25.175 & 97.2748 \\
\hline 5 & 26.060 & 0.3904 \\
\hline 6 & 27.018 & 0.6970 \\
\hline
\end{tabular}




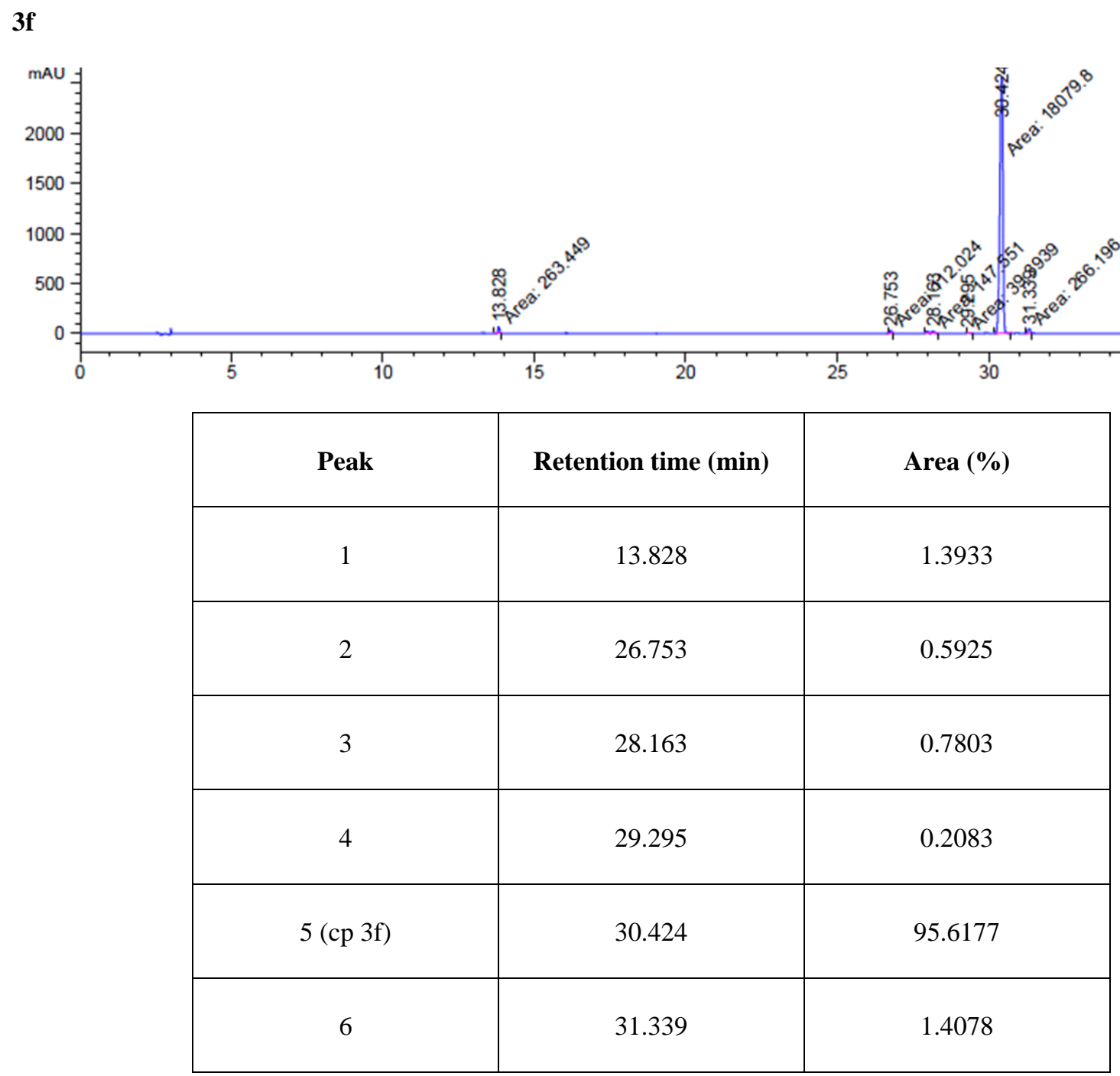

3g

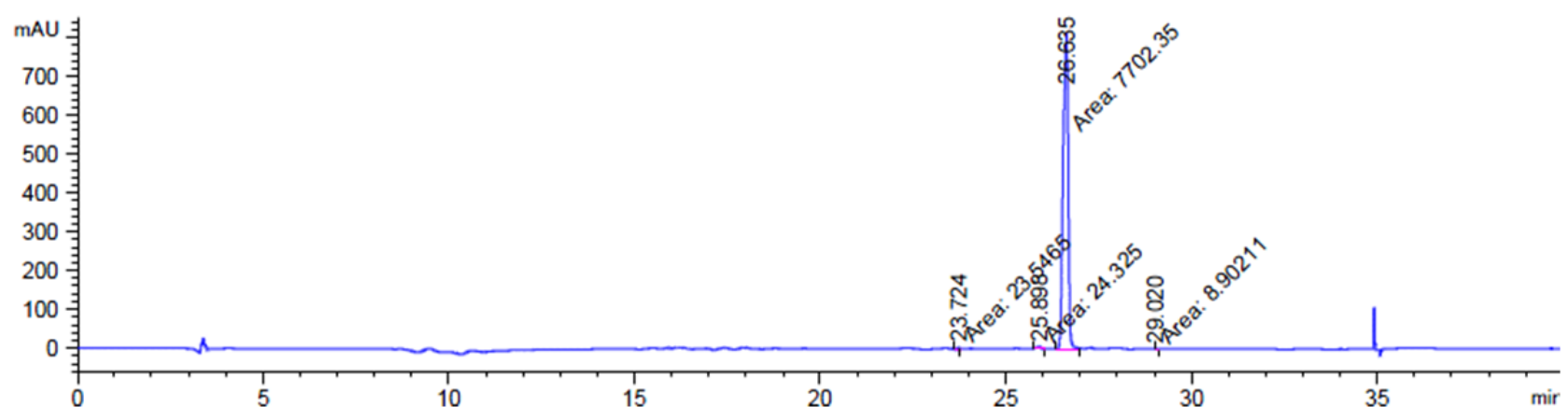




\begin{tabular}{|c|c|c|}
\hline Peak & Retention time (min) & Area (\%) \\
\hline 1 & 23.724 & 0.3035 \\
\hline 2 & 25.898 & 0.3135 \\
\hline $3($ ср 3g) & 26.635 & 99.2683 \\
\hline 4 & 29.020 & 0.1147 \\
\hline
\end{tabular}

6

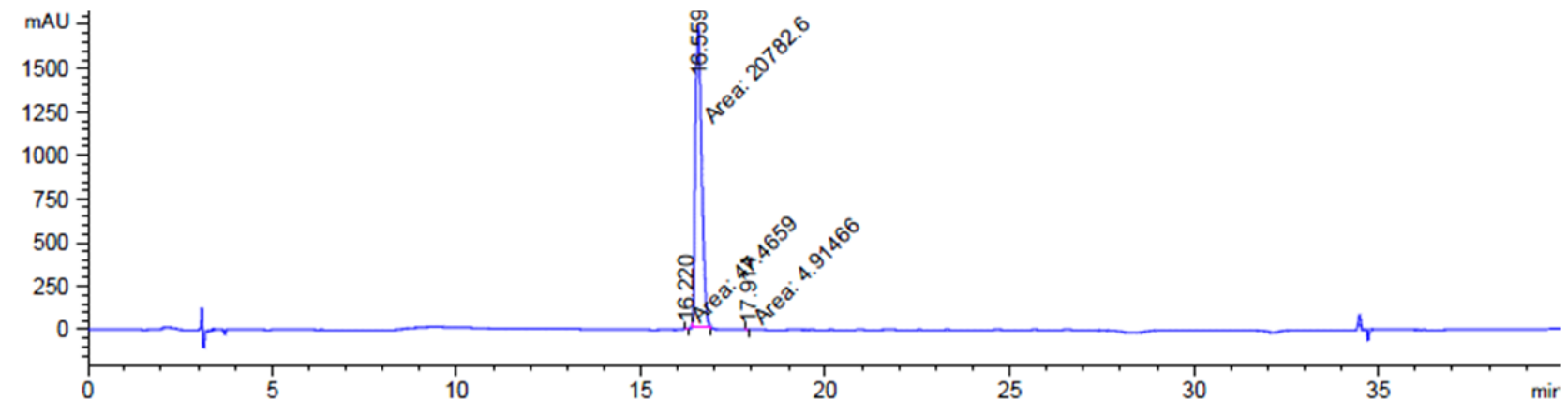

\begin{tabular}{|c|c|c|}
\hline Peak & Retention time (min) & Area (\%) \\
\hline 1 & 16.220 & 0.2287 \\
\hline $2(\mathrm{cp} \mathrm{6)}$ & 16.559 & 99.7486 \\
\hline 3 & 17.914 & 0.0236 \\
\hline
\end{tabular}

11

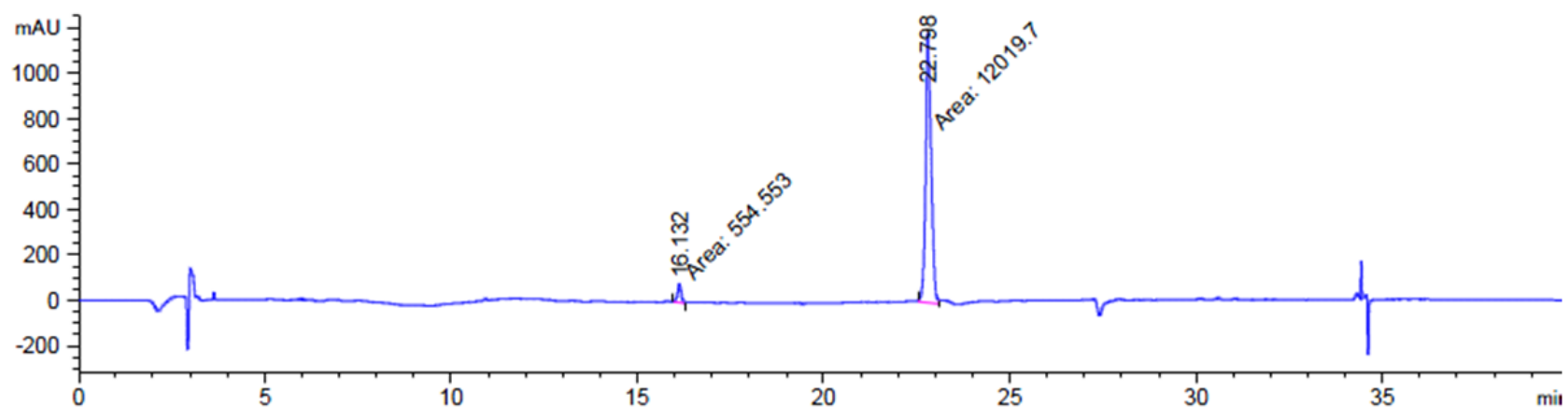




\begin{tabular}{|c|c|c|}
\hline Peak & Retention time (min) & Area (\%) \\
\hline 1 & 16.132 & 4.4102 \\
\hline 2 (ср 11) & 22.798 & 95.5898 \\
\hline
\end{tabular}

12

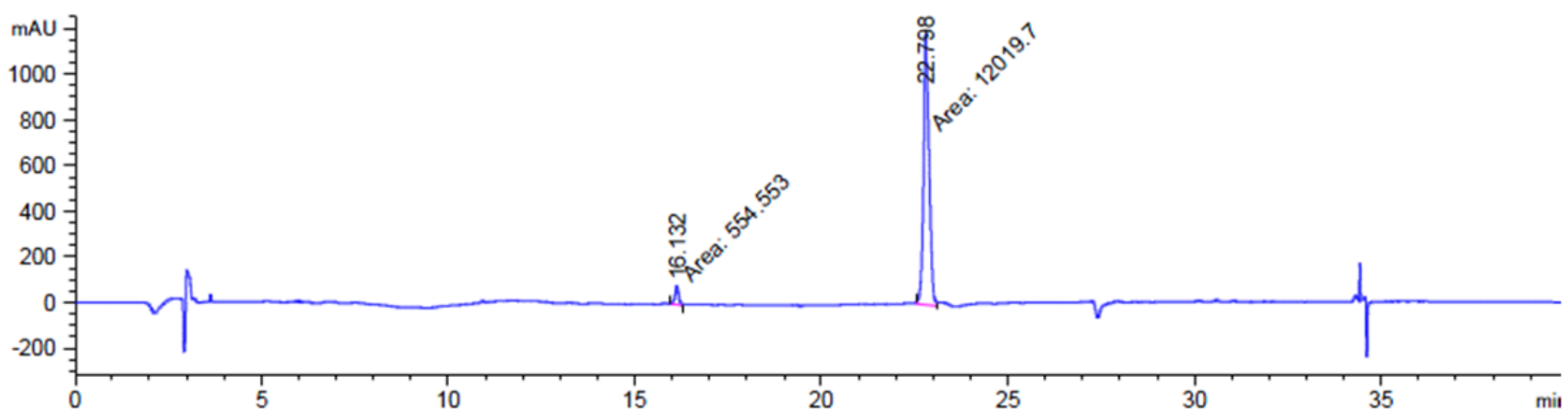

\begin{tabular}{|c|c|c|}
\hline Peak & Retention time (min) & Area (\%) \\
\hline 1 & 13.342 & 1.1369 \\
\hline 2 (ср 12) & 23.711 & 98.8631 \\
\hline
\end{tabular}

\section{Supplementary references}

(1) Thomas, A. M.; Asha, S.; Sindhu, K. S.; Anilkumar, G. A General and Inexpensive Protocol for the Cu-Catalyzed C - S Cross-Coupling Reaction between Aryl Halides and Thiols. Tetrahedron Lett. 2015, 56, 6560-6564.

(2) Biscoe, M. R.; Fors, B. P.; Buchwald, S. L. A New Class of Easily Activated Palladium Precatalysts for Facile C - N Cross-Coupling Reactions and the Low Temperature Oxidative Addition of Aryl Chlorides. J. Am. Chem. Soc. 2008, 130, 6686-6687.

(3) Vicker, N.; Xiangdong, S.; Ganeshapillai, D.; Purhoit, A.; Reed, M. J.; Potter, B. WO2005042513A1. 2005. 
(4) Lancelot, J.; Caby, S.; Dubois-Abdesselem, F.; Vanderstraete, M.; Trolet, J.; Oliveira, G.; Bracher, F.; Jung, M.; Pierce, R. J. Schistosoma Mansoni Sirtuins: Characterization and Potential as Chemotherapeutic Targets. PLoS Negl. Trop. Dis. 2013, 7, 1-13.

(5) (48) Lancelot, J.; Cabezas-Cruz, A.; Caby, S.; Marek, M.; Schultz, J.; Romier, C.; Sippl, W.; Jung, M.; Pierce, R. J. Schistosome Sirtuins as Drug Targets. Future Med. Chem. 2015, 7, 765-782. 
Other files 